\title{
Cathodoluminescent screen imaging system for seeded blob detection in Toroidal Plasma Experiment
}

\author{
P. Micheletti, ${ }^{*}$ M. Baquero-Ruiz, F. Manke, I. Furno, P. Ricci, and A. Fasoli \\ École Polytechnique Fédérale de Lausanne (EPFL), Swiss Plasma Center (SPC), CH-1015 Lausanne, Switzerland. \\ P. Bowen, C. Morais, and W. Zhao \\ École Polytechnique Fédérale de Lausanne (EPFL), Powder Technology Laboratory (LTP), CH-1015 Lausanne, Switzerland
}

(Dated: April 28, 2020)

\begin{abstract}
We designed and built a diagnostic based on a cathodoluminescent screen for detection of turbulent plasma structures with high spatial resolution. The screen is coated with a low threshold energy cathodoluminescent powder that emits light when exposed to a plasma. The emitted light is imaged with a fast frame camera combined with an image intensifier and an optical band pass filter. The diagnostic is used to study turbulent structures and seeded blobs. The results are analyzed with pattern recognition algorithms to track the turbulent structures and study their evolution in time.
\end{abstract}

\section{INTRODUCTION}

Plasma turbulent structures and blobs can efficently convect particles and heat across magnetic field lines [1-5]. An effective diagnosis of turbulent structures is therefore a key point to understanding heat and particle transport dynamics. Basic plasma devices such as the TORoidal Plasma EXperiment (TORPEX) [6] aim to investigate turbulence and turbulent structures. Diagnostic methods are often based on plasma light emission or Langmuir probe arrays, which lack spatial resolution or are based on detection of line integrated quantities [7]. For instance the HEXTIP-U diagnostic, which consists in two distinct two-dimensional (2D) arrays of 95 Langmuir probes (LP), is used in TORPEX to infer 2D, and even $3 \mathrm{D}$, plasma properties. Although high resolution could be achieved in principle with denser arrays of LPs, the distance between each probe cannot be decreased arbitrarily since overlap of plasma sheaths of different LPs must be avoided. At the same time, the LP density is limited by the perturbation of the array on the plasma, in particular the current drawn by the LPs must be negligible with respect to the charged particle generation rate [8]. In practical terms, this limits the separation between probes to $\sim 1 \mathrm{~cm}$ when covering large sections of the poloidal cross section [8].

Cathodoluminescent phosphor coatings have a local response to streams of electrons. For that reason they have been used to image 2D structures of neutral and non-neutral plasma [9-11]. ZnO:Zn powder, in particular, shows properties such as vacuum compatibility and the ability to withstand high current densities [12] that make it suitable for fusion applications, including the investigation of magnetic topology in stellarators $[13,14]$. It is also an interesting alternative to resolve time evolution of plasma structures with features at the millimeter level. This opens the possibility of studying blob phenomena such as secondary instabilities and fragmentation, which may determine their size and constrain its overall radial velocity [15].

*paolo.micheletti@epfl.ch
In this paper, we describe the design of a CathodoLuminescent Screen (CLS)-based imaging system and its implementation in TORPEX. A particular emphasis is given to the discussion on the cathodoluminescent coating process and the enhancement of the imaging system acquisition rate and noise rejection (Sec. II), which are essential to obtain fast and high quality images. To distinguish the evolution and propagation of turbulent structures with respect to other phenomena, a local plasma production system is designed that allows directly producing seeded blobs (Sec. III). Seeded turbulent structures are characterized with the CLS revealing their features with remarkable spatial resolution. The results, shown in Sec. IV, are followed by a discussion and an outlook of potential studies enabled by the CLS (Sec. V).

\section{TORPEX AND THE CLS DIAGNOSTIC}

TORPEX is a toroidal plasma device located at the Swiss Plasma Center (SPC) in Lausanne, Switzerland, used in basic studies of magnetized plasmas of hydrogen, argon or other gases [6]. The experimental vessel has a major radius $R=1 \mathrm{~m}$ and a minor radius $a=20 \mathrm{~cm}$. The discharges are obtained by introducing the corresponding gas at a pressure $p \approx 10^{-4}-10^{-5} \mathrm{mbar}$ and injecting MicroWaves (MW) at a frequency $f_{M W}=2.45 \mathrm{GHz}$, which are absorbed at the electron-cyclotron and upper-hybrid resonances [16]. When used in the Simple Magnetized Torus (SMT) configuration, the magnetic field typically has a dominant toroidal component $B_{\phi} \approx 70 \mathrm{mT}$ and a small vertical component $B_{z} \leqslant 5 \mathrm{mT}$. Plasmas with densities $n \approx 10^{15}-10^{17} \mathrm{~m}^{-3}$, temperatures $T_{e} \approx 5 \mathrm{eV}$ and plasma potentials $V_{p l} \approx 10-20 \mathrm{~V}$ are obtained, which exhibit turbulent features and are well suited for studies of turbulent plasma structures $[6,8,17,18]$.

An important role is played by the HEXTIP-U [8] LP arrays, that are located at two diametrically opposed toroidal angles (north-west (NW) and south-east (SE) arrays respectively). HEXTIP-U allows to perform 2D measurements of saturation current $\left(I_{\text {sat }}\right)$ or floating potential $\left(V_{\text {float }}\right)$. The sampling rate can go up to $250 \mathrm{kHz}$, thus guaranteeing a time resolution which has been seen to be appropriate to observe 
a)

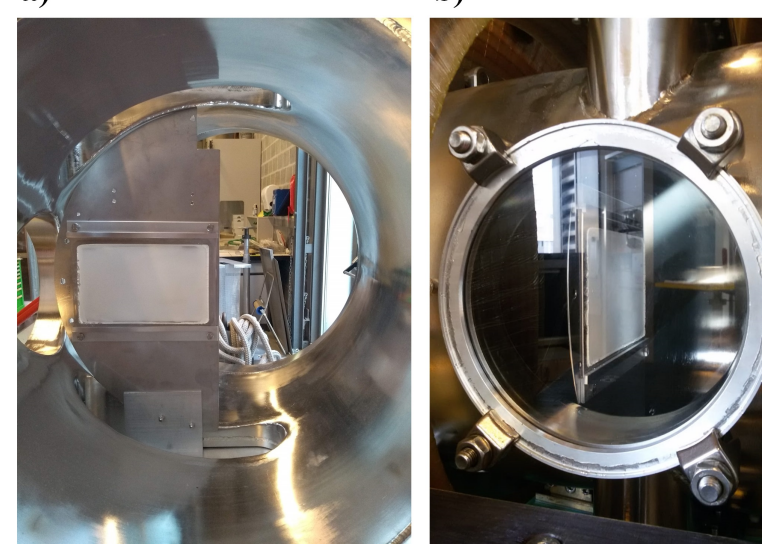

FIG. 1. Stainless steel limiter with cathodoluminescent coating. (a) Placement of limiter in a mock-up TORPEX sector. The limiter covers the Low Field Side (LFS) half of the poloidal cross section. (b) CLS after installation and under high vacuum seen from a transparent flange.

the evolution of individual plasma structures [5, 6, 8, 19-22]. Spatial resolution, on the contrary, has been has proven to be insufficient in certain experiments where structures can have sizes smaller than the spacing between the probes or present feature that cannot be properly resolved.

The CLS diagnostic aims to overcome the resolution limit of HEXTIP-U. It consists of a rectangular stainless steel plate ( $15.5 \mathrm{~cm}$ wide and $9.5 \mathrm{~cm}$ heigh) coated with a low threshold energy $\left(E_{t h}=3.0 \mathrm{eV}\right)$ and short persistence time $(\approx 1 \mu \mathrm{s})$ cathodoluminescence powder of $\mathrm{ZnO}: \mathrm{Zn}$ phosphor P-24 [23]. This material emits light at $\sim 500 \mathrm{~nm}$ when its surface is exposed to a plasma, with luminance proportional to $n_{e} T_{e}^{2.25}$ [9]. This relation holds whenever the coating is electrically floating. In this case the flux of electrons on the screen, hence the luminescence, is independent of the plasma potential [9].

The powder [23], with particle size $\sim 1 \mu \mathrm{m}$, is deposited on the steel plate with a sedimentation method. The plate is placed in a mould and a liquid suspension is poured on it. The liquid is then slowly evaporated at room temperature in a humid atmosphere (relative humidity $>95 \%$ ). A uniform coating of phosphor powder is left on the steel plate. The liquid medium consists in aqueous solution with $0.25 \mathrm{wt} . \%$ polyacrylic acid allowing for a better dispersion. A solution to powder weight ratio of $2: 1$ is used. The process is completed by introducing the plate in an oven at $60^{\circ} \mathrm{C}$ in air to complete the evaporation of the solution and to stabilize the coating [24]. The coated screen is then placed on a holder fixed to a stainless steel limiter installed on the low field side (LFS) of TORPEX (Fig. 1). Although the limiter plate is grounded, as it is directly connected to the vessel, the particular deposition technique yields a coating with very high resistance $\left(R_{\text {screen }}>10^{6} \Omega\right)$, thus guaranteeing that the exposed surface is effectively floating.

The screen is imaged directly through a transparent flange with an optical set-up (Fig. 1, 2). A black box with mirrors, placed in front of the window, improves the viewing angle of
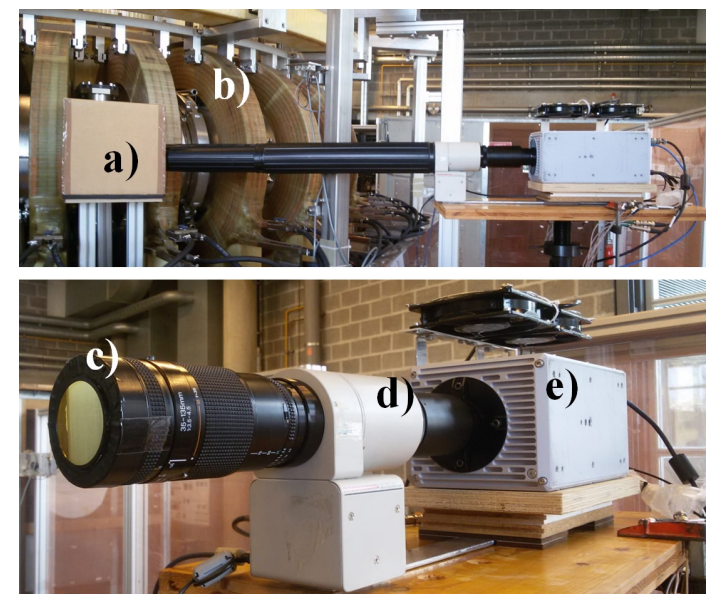

FIG. 2. Imaging system and its different components. (a) Dark box with mirrors to improve the view angle on the coating. (b) TORPEX vacuum vessel and toroidal magnetic field coils. (c) Optical passband filter. (d) Image Intensifier Unit. (e) FastCam.

the screen and blocks the external light. To reduce unwanted reflections, the mirrors have first-surface coatings. Plasma luminosity is filtered out with a band-pass optical filter centred at $510 \pm 25 \mathrm{~nm}$. The pass band of the filter matches the expected emission peak of the coating, and is at the same time far from any strong emission lines of $\mathrm{H}_{2}$ and Ar. The signal is amplified with a Hamamatsu C10880-03F Image Intensifier Unit (IIU), to obtain an image with good intensity even at high frame rates, hence low integration times. The images are acquired with a Photron FastCam-APX RS model 250k [25] used with a frame rate $f_{a q}=100 \mathrm{kfps}$ (kilo-frames per second). The value of $f_{a q}$ is the result of a trade-off between time resolution of the measurement and the image resolution, which is set, on average, $\approx 1 \mathrm{pixel} / \mathrm{mm}$. Indeed the camera allows for higher frame rates but at the cost of lower spatial resolution. To synchronize the image acquisition with HEXTIP-U, the two diagnostics are fed with the same clock signal. However, to avoid losing the temporal resolution of HEXTIP-U, a simple frequency divider is introduced to obtain a camera frame rate of $100 \mathrm{kfps}$ and a HEXTIP-U sampling rate of $200 \mathrm{kHz}$.Once the images are taken, a post processing step is performed to correct the view angle of the CLS. The area of the screen is cropped and projected to obtain a front view of the coated part.

\section{LOCAL BREAKDOWN MECHANISM FOR SEEDED BLOB PRODUCTION}

A system for local breakdown of $A r$ has been recently implemented in TORPEX using an In-Vessel Coil (IVC). The method is similar to the one used in previous works [26]. The toroidal field in TORPEX is approximately proportional to $1 / R$. The EC resonance is therefore expected to occur at specific values of $R_{\text {res }}$ where $B\left(R_{\text {res }}\right)$ fulfils the condition $f_{M W}=$ $(q / m) \times\left(B_{0} / R_{r e s}\right)$. If $B_{0}$ is chosen such that $R_{r e s}<R-a$, there is no microwave absorption in the chamber and, thus, 
a) Coil

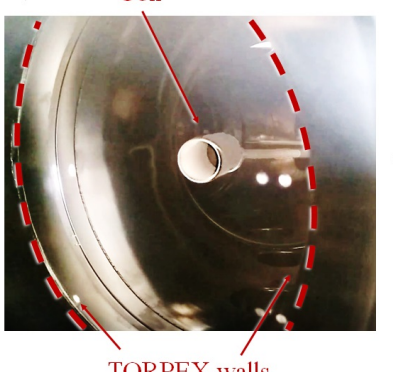

b)

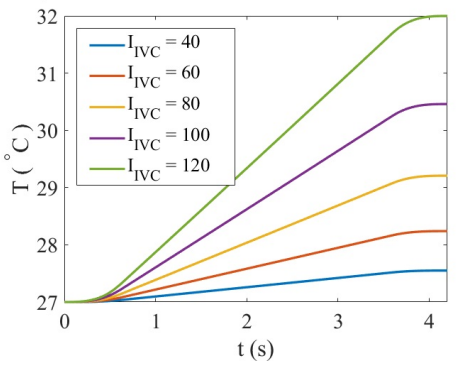

a) HEXTIP-U SE (t=166.194ms $)$

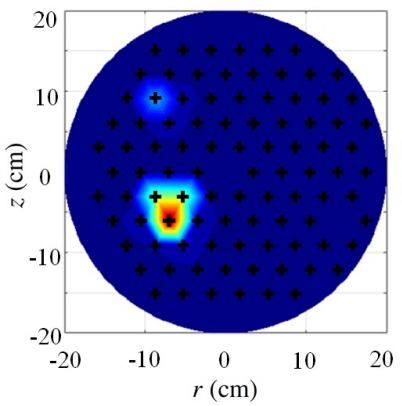

b) HEXTIP-U NW (t=166.194ms)

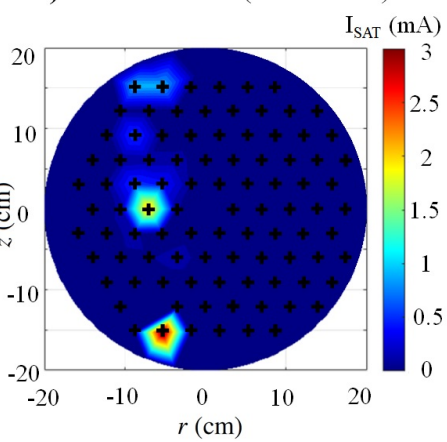

FIG. 3. (a) IVC inside TORPEX held by a ceramic arm, (b) Study of the IVC temperature increase due to ohmic heating. The IVC current $\left(I_{I V C}\right)$ is ramped-up from 0 to the nominal value $([40,60,80,100,120]$ A respectively) in $0.6 \mathrm{~s}$, kept constant for $3 \mathrm{~s}$ and then ramped down to 0 .

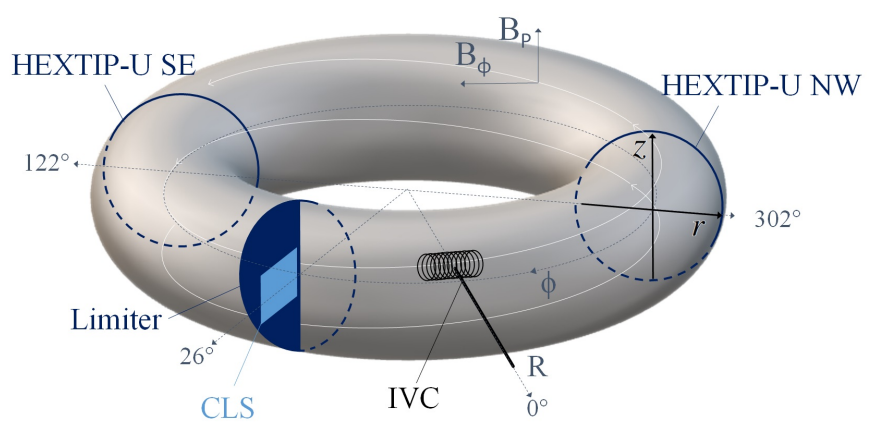

FIG. 4. Schematic representation of the experimental setup, with a particular focus on the position of HEXIP-U SE, HEXTP-U NW, the CLS, the IVC. An example of field line in SMT configuration is depicted in white.

no plasma is generated. However, the introduction of the IVC allows to modify the toroidal field distribution such that it locally reaches the value required for the electron-cyclotron resonant breakdown $\left(B_{\text {res }}\right)$. In this way the $2.45 \mathrm{GHz}$ microwaves can be absorbed only in the volume inside the coil. Thus, by injecting a pulse of microwaves in the vessel, it is possible to generate seeded blobs. Stationary filaments can also be generated keeping the microwave power constant.

The IVC consists of a copper coil with radius $R=2.4 \mathrm{~cm}$, length $l=10 \mathrm{~cm}$ and 35 windings, coated with a liquid ceramic to insulate it from the plasma (Fig. 3). The geometrical dimensions of the solenoid are chosen to maximize the field strength achievable while keeping the temperature increase, due to ohmic heating, under the reasonable limit of $\sim 50^{\circ} \mathrm{C}$ (Fig. 3). The coil is held in place by a ceramic arm which can be moved radially.

Preliminary tests in the SMT configuration are performed to demonstrate the effective production of single plasma filaments. In these experiments only HEXTIP-U is used to diagnose the plasma profile, while the CLS is not present in the chamber. The IVC is placed on the high field side at $z=0 \mathrm{~cm}, r=-8 \mathrm{~cm}$, ' $r$ ' and ' $z$ ' being the usual horizontal

FIG. 5. Interpolated $I_{S A T}$ distribution in shot \# 70703 measured with HEXTIP-U (a) at the NW array and (b) the SE array. The measurement is taken $(250 \pm 10) \mu \mathrm{s}$ after the beginning of the MW pulse. Black crosses mark the position of the individual LPs. The color scale of both plots is indicated in the bar on the right.

and vertical coordinates in the poloidal cross section (Fig. 4). Considering the IVC toroidal position as $\phi=0^{\circ}$ and proceeding clockwise, HEXTIP-U SE and HEXTIP-U NW are placed at $\phi=122^{\circ}$ and $\phi=302^{\circ}$ respectively (Fig. 4). Pulses of microwaves with a peak power of $P_{M W} \approx 6 \mathrm{~kW}$ and length $\Delta t_{M W} \leq 1 \mathrm{~ms}$ are used to achieve local breakdown of Ar gas. This choice of gas leads to appreciable breakdown in our experiments, possibly due to its high ionization cross section. The spatial distribution of the ion saturation current $\left(I_{S A T}\right)$ is measured with HEXTIP-U applying a negative bias $(-42 \mathrm{~V}$, see Ref. [8]). An example of a plasma filament obtained with $B_{\phi}(r=0)=64 \mathrm{mT}, B_{z}(r=0, z=0)=1.8 \pm 0.12 \mathrm{mT}$ and IVC current $I_{I V C}=58 \mathrm{~A}$ is reported in Fig. 5. The profiles of $I_{S A T}$ measured with HEXTIP-U show that the plasma is effectively arranged in a field-aligned structure, well confined in the poloidal plane and extends along the field lines. Once produced, the structure propagates radially and expands until it reaches the wall on the LFS. An example of the time evolution of a seeded structure obtained with the same parameters is shown in Fig. 6.

\section{EXPERIMENTAL TESTS}

The CLS is used in two different experimental setups. First we use a simple SMT configuration with no IVC. In this configuration a slab-shaped plasma is obtained which has been seen to produce blobs by an ideal interchange mode [5, 8, 19-21]. In particular, a magnetic configuration with $B_{\phi}=76.7 \mathrm{mT}$ and $B_{z}=1.3 \mathrm{mT}$ is used with constant microwave power of $600 \mathrm{~W}$. In this way we obtain an $\mathrm{H}$ plasma with a turbulent edge close to the edge of the limiter. This configuration is well adapted to image plasma turbulent structures as they propagate towards the LFS. Plasma density in the whole poloidal section is monitored with HEXTIP-U, while turbulent structure behavior is captured with the CLS and the imaging system. In this experiment, 1000 images at $100 \mathrm{kfps}$ are acquired, using an amplification voltage at the IIU of 

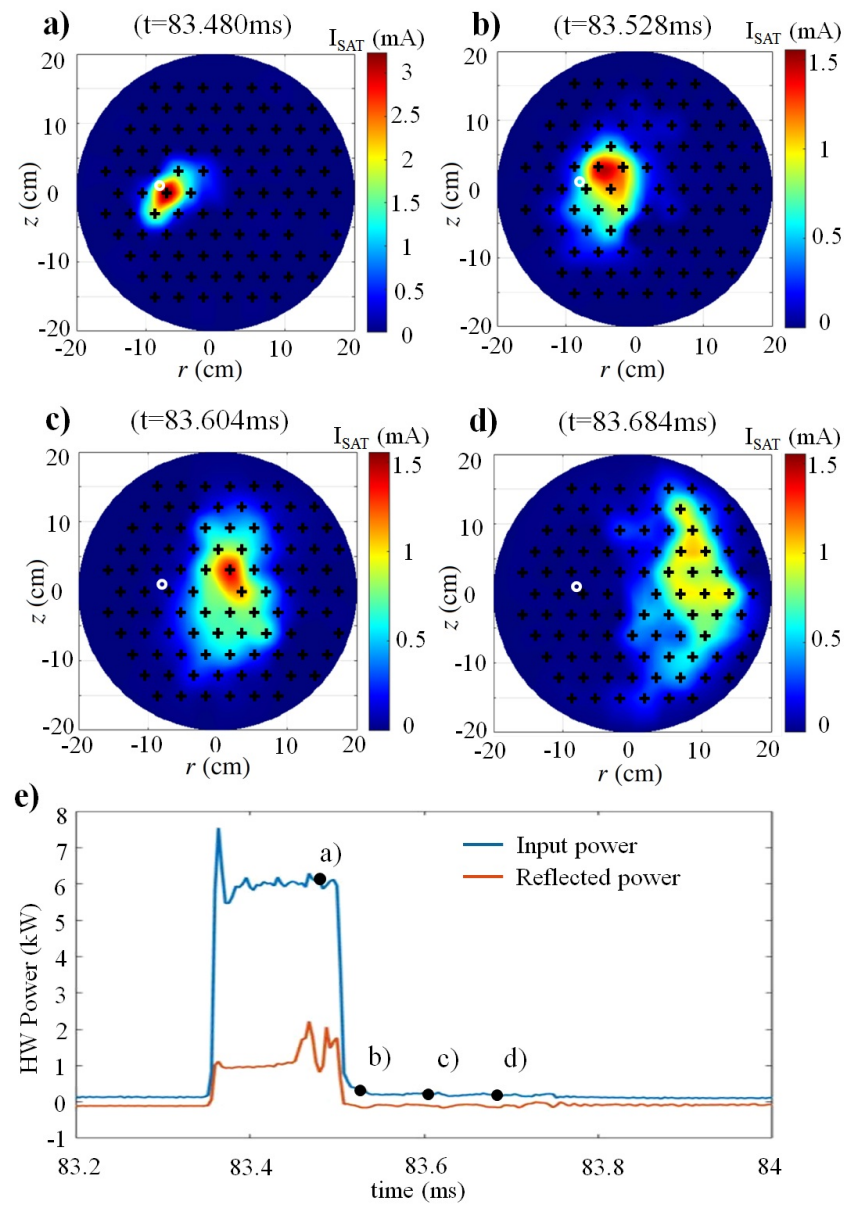

FIG. 6. Interpolated $I_{S A T}$ distribution in shot \# 70864 measured with HEXTIP-U SE at a) $t=83.480 \mathrm{~ms}$, b) $t=83.528 \mathrm{~ms}$, c) $t=$ $83.604 \mathrm{~ms}$ and d) $t=83.684 \mathrm{~ms}$. e) Measurement of the injected and reflected microwave power. The black ' $\bullet$ ' indicates the positions in time of the images above. The white circle indicate the position of the IVC projected on the HEXTIP-U SE plane

$850 \mathrm{~V}$, and setting the gating time, i.e. the integration time per frame, at $4 \mu \mathrm{s}$ [24].

Single structures propagating towards the low field side are observed in this configuration. An example obtained with $B_{\phi}=76.7 \mathrm{mT}$ and $B_{z}=1.3 \mathrm{mT}$ is shown in Fig. 7. The structure, $\approx 2 \mathrm{~cm}$ in size, propagates vertically and radially while becoming elongated.

The quality of the measurements can be assessed comparing the signal obtained in this configuration with a reference. A configuration with a lower toroidal field $\left(B_{\phi}=71.9 \mathrm{mT}\right)$, i.e. where plasma production occurs far from the CLS, is used for this purpose. In this case the CLS signal is entirely given by the UV radiation produced by the background plasma. The quality of the images is therefore evaluated comparing these two configurations (Fig. 8). In the first scenario (Fig. 8 a-b ) the regions affected by turbulent structures show a high temporal average and high standard deviation (STD) due to the high variability of the signal. On the other hand, the signal relative to the second scenario (Fig. $8 \mathrm{c}-\mathrm{d}$ ) has a much lower
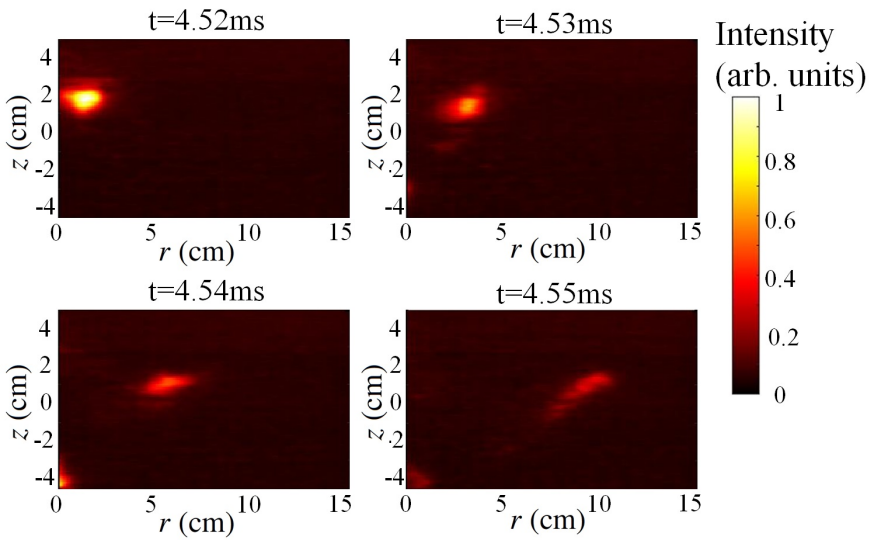

FIG. 7. Single images of the CLS exposed to a $\mathrm{H}$ plasma (shot \# 69490) showing a single plasma structure. The image intensity is normalized to 1 dividing by the maximum intensity on time and space. The colorscale, shared by the four images, shows the intensity in arbitrary units and it is reported on the right.
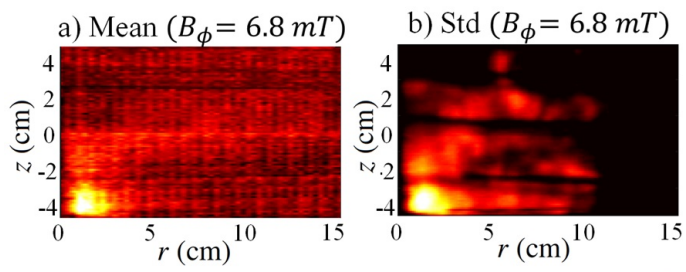

Intensity

(arb. units)
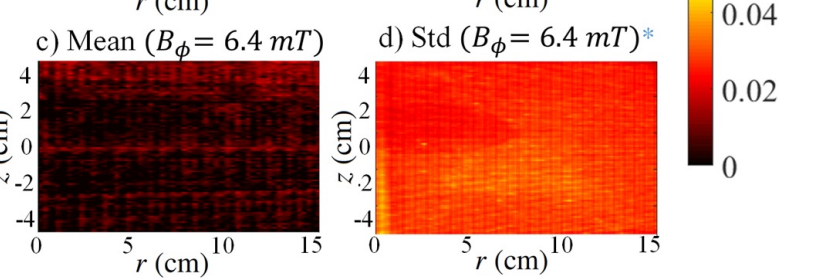

FIG. 8. Statistics of the images of the CLS exposed to a $\mathrm{H}$ plasma (shot \# 69490 a) and b), shot \# 69481 c) and d) ). The image intensity is normalized to 1 upon dividing by the maximum intensity observed taking into account both shots. The coordinates are assigned according to the screen position inside TORPEX vessel. The colorscale, shared by the four images, shows the intensity in arbitrary units and it is reported on the right, for the image d) the colorscale is the same but multiplied by $10^{-1}$.

time average. This indicates that the signal given by the turbulent structures passing on the CLS is effectively much higher than the background. At the same time Fig. $8 \mathrm{~d}$ ) indicates that the fluctuations of the signal are small even when the background plasma and the UV radiation are the only excitation sources of the CLS. These observations confirm that we can effectively track plasma structures frame by frame without the need of averaging. This first experiment demonstrates the effectiveness of the CLS to reveal and track plasma turbulent structures with high spatial resolution.

In the second experiment, the CLS is used to study the propagation of turbulent structures which are produced in Ar using the IVC. The CLS is placed close to the IVC at $\phi=26^{\circ}$ (Fig. 4). This configuration allows the measurements to be 
much less sensitive to HEXTIP-U shadowing, since plasma production occurs close to the CLS, which is placed as far as possible from the LP array.

We investigate several configurations with different positions of the IVC $\left(r_{I V C} \in[-8,-2] \mathrm{cm}\right)$ and values of vertical field strength $\left(B_{z} \in[0,2.25] \mathrm{mT}\right)$. To achieve local breakdown microwaves are injected in pulses with $\Delta t_{\text {pulse }} \approx 3.6 \mathrm{~ms}$ and a repetition rate of $110 \mathrm{~Hz}$. The peak heating power $P_{M W}$ ranges between $1.5 \mathrm{~kW}$ and $2 \mathrm{~kW}$ and is tuned in each case depending on the magnetic configuration. The distance measured along the field lines between the IVC and the top and bottom surfaces of TORPEX, i.e. the connection length of the plasma structure, depends on the field strength. Thus a variation of vertical field results in a variation of the energy required for breakdown. Therefore the product $\Delta t_{p u l s e} P_{M W}$ must be carefully tuned to produce a structure and then let it propagate without injecting more power.

In Fig. 9 a discharge, obtained with $B_{\phi}=64 \mathrm{mT}$ and $B_{z}=$ $0 \mathrm{mT}$, shows the radial propagation of a seeded blob. The $I_{S A T}$ profiles measured with HEXTIP-U show that the structure is initially fully symmetric, while the symmetry is lost as the structure propagates radially. This is likely due to the presence of the CLS which is expected to perturb the plasma. However the fact that the three diagnostics agree on the propagation velocity of the structure suggests that its spatial coherence is maintained during the propagation. Although the $I_{S A T}$ profiles show an almost homogeneous distribution of the filament cross section, structures with a size of few centimeters are revealed by the CLS-based imaging system that cannot be identified by HEXTIP-U due to its lower resolution. These structures seem to be the result of a fragmentation of the seeded blob front. A pattern recognition algorithm is implemented to identify the structures in the CLS images and in $I_{S A T}$ profiles to track their trajectory and velocity in time. In order to compute these quantities the frame of reference of each structure is defined as its mean position weighted by the intensity. The structure trajectory is defined as the position of its frame of reference in time and it is of the order of $\sim 100 \mathrm{~m} / \mathrm{s}$. It is also possible to decouple the structure propagation from other phenomena such as the evolution of its intensity distribution.

We performed a statistical analysis on the data, relating the propagation velocity perpendicular to the magnetic field lines, to the vertical size of the structure. Fig. 10 shows the probability distribution function (PDF) of these quantities for each turbulent structure revealed by HEXTIP-U and the CLS diagnostics, obtained in the experiments described above, i.e. for the whole set of parameters investigated $\left(r_{I V C} \in[-8,-2] \mathrm{cm}\right.$, $\left.B_{z} \in[0,2.25] \mathrm{mT}, P_{M W} \in[1.5,2] \mathrm{kW}\right)$. The PDF is computed discretizing the two variables and evaluating the frequency of occurrence of each pair of values, the distribution obtained in this way is then divided by its integral. The perpendicular velocity $v_{\perp}$ and perpendicular size $a_{\perp}$ are normalized, as in [22], using

$$
v^{*}=\left(\frac{2 L \rho_{s}^{2}}{R^{3}}\right)^{1 / 5} c_{s}, \quad a^{*}=\left(\frac{4 L^{2}}{\rho_{s} R}\right)^{1 / 5} \rho_{s},
$$

where $R$ is TORPEX major radius, $L \approx 2 \pi R$ is the connec- tion length, nearly constant in this case due to the presence of the limiter, $\rho_{s} \approx \sqrt{T_{e} m_{i}} / e B$ is the ion sound Larmor radius and $c_{s} \approx \sqrt{T_{e} / m_{i}}$ is the ion sound speed. A typical electron temperature of $T_{e}=3.5 \mathrm{eV}$ [22] is assumed. Typical values of these quantities, e.g. the ones obtained for shot \# 71213, are $v^{*}=1.2 \mathrm{~km} / \mathrm{s}$ and $a^{*}=13 \mathrm{~cm}$. Hence, it is possible to compare the PDF of $\tilde{v}_{\perp}=v_{\perp} / v^{*}$ and $\tilde{a}_{\perp}=a_{\perp} / a^{*}$ with a blob velocity scaling law [22]:

$$
\tilde{v}_{\perp}=\frac{\sqrt{2 \tilde{a}_{\perp}} \delta n / n}{1+\sqrt{2} \tilde{a}_{\perp}^{5 / 2}+\tilde{\eta} \sqrt{\tilde{a}_{\perp}}},
$$

where $\delta n / n$ is the fluctuation density over the background density and $\tilde{\eta}$ is the normalized ion-neutral friction here assumed to be zero by neglecting the ion-neutral collisions. A constant temperature with a typical value of $T_{e}=3.5 \mathrm{eV}$ [22] is assumed, hence the effects of its fluctuation are neglected. The corrections that may be associated to this approximation will be studied in future work.

Thanks to the CLS a whole new class of structures can be identified. The PDF of these structures (Fig. 9b) seems to follow the prediction of the velocity scaling law, confirming its validity for $\tilde{a}_{\perp} \leq 0.1$. A slight deviation from the predictions can be identified for structures with $\tilde{a}_{\perp} \leq 0.05$. This may indicate the presence of different mechanisms involved in their propagation such as finite Larmor radius effects, which will be matter of further studies.

\section{v. CONCLUSIONS}

In this paper, we described the design, implementation and operation of a CLS based imaging system. The system consists of a cathodoluminescent coating imaged with a fast camera and a IIU which allow to acquire images with a frame rate of up to $100 \mathrm{kfps}$. With appropriate optical filters, we obtain images with good signal to noise ratio of turbulent plasmas in the SMT configuration. The diagnostic is also used in combination with a plasma filament seeding system (the IVC), to track the evolution of turbulent structures with remarkable spatial resolution. Data obtained with the CLS and with HEXTIP-U have been shown to offer complimentary information that allows to gain insight about the propagation and evolution of seeded turbulent structures and to identify features with sizes down to $\sim 1 \mathrm{~mm}$. A pattern recognition algorithm is used to extract basic properties of the structures.

The new diagnostics opens the possibility of studying phenomena related to blob fragmentation and possible development of secondary instabilities. Future work will be focused on the investigation of the nature of the structures revealed by the cathodoluminescent limiter and the mechanisms involved in their formation and propagation. Upgrades in the system to seed plasma structures are envisioned to achieve a finer control of the size and allow breakdown in other gasses such as $\mathrm{H}_{2}$ and $\mathrm{He}$, more relevant for fusion applications. Finally further experiments are planned to analyze the effects of different magnetic configurations on structure propagation. 

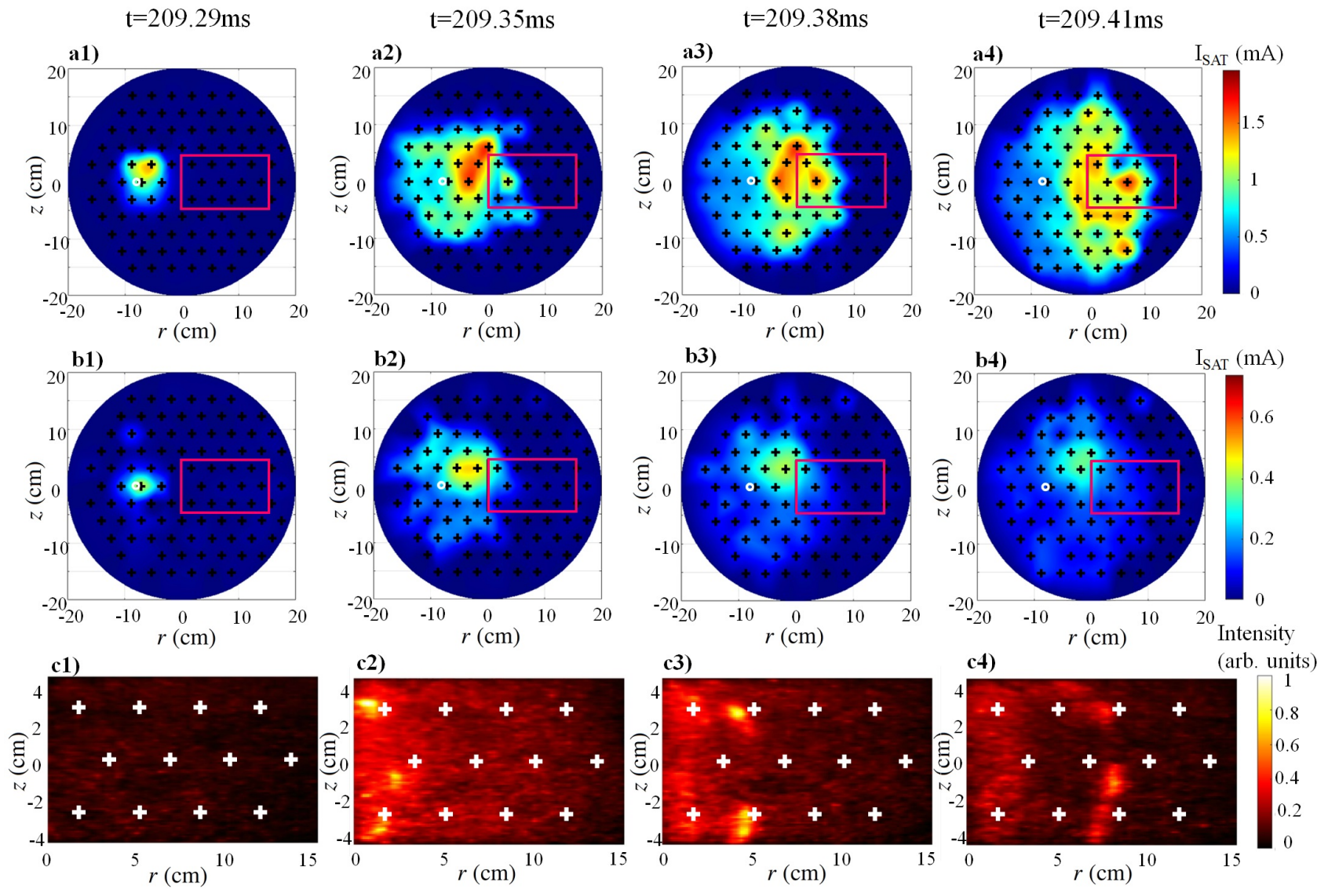

FIG. 9. Interpolated $I_{S A T}$ distribution in shot \#71213 measured with HEXTIP-U a) SE and b) NW array and image of the CLS captured with the optical set-up (c). The red rectangle on the interpolated $I_{S A T}$ plots indicate the position of the CLS projected on the HEXTIP-U array toroidal position. The white crosses on the CLS images indicate the projected position of the probes of HEXTIP-U enclosed in the screen area. In this case the projection of the two arrays coincide since $B_{z}=0 \mathrm{mT}$. Each column is relative to a different instant: 1) $t=209.29 \mathrm{~ms}, 2$ ) $t=209.35 \mathrm{~ms}, 3) t=209.38 \mathrm{~ms}$ and 4) $t=209.41 \mathrm{~ms}$. The plots of each line share a common color scale which is reported on the right.

\section{ACKNOWLEDGEMENT}

This work has been carried out within the framework of the EUROfusion Consortium and has received funding from the
Euratom research and training programme 2014 - 2018 and 2019 - 2020 under grant agreement No 633053. The views and opinions expressed herein do not necessarily reflect those of the European Commission. This work was supported in part by the Swiss National Science Foundation.
[1] S. J. Zweben, Phys. Fluids 28, 974 (1985).

[2] B. Labit, A. Diallo, A. Fasoli, I. Furno, D. Iraji, S. Müller, G. Plyushchev, M. Podestà, F. Poli, P. Ricci, C. Theiler, and J. Horaček, Plasma Phys. Control. Fusion 49, B281 (2007).

[3] F. Riva et al., Plasma Phys. Control. Fusion 58, 044005 (2016).

[4] R. Sánchez, B. P. van Milligen, D. Newman, and B. Carreras, Phys. Rev. Lett. 90, 185005 (2003).

[5] I. Furno, B. Labit, M. Podestà, A. Fasoli, S. H. Müller, F. M. Poli, P. Ricci, C. Theiler, S. Brunner, A. Diallo, and J. Graves, Phys. Rev. Lett. 100, 055004 (2008).

[6] I. Furno, A. Burckel, L. Federspiel, A. Fasoli, K. Gustafson, D. Iraji, B. Labit, J. Loizu, G. Plyushchev, P. Ricci, C. Theiler, A. Diallo, S. H. Mueller, M. Podestà, and F. Poli, Plasma Phys. Control. Fusion 52, 124020 (2010).
[7] S. J. Zweben, J. A. Boedo, O. Grulke, C. Hidalgo, B. LaBombard, R. J. Maqueda, P. Scarin, and J. L. Terry, Plasma Phys. Control. Fusion 49, S1 (2007).

[8] M. Baquero-Ruiz, F. Avino, O. Chellai, A. Fasoli, I. Furno, R. Jacquier, F. Manke, and S. Patrick, Rev. Sci. Instrum. 87, 113504 (2016).

[9] A. Liebscher, S. Luckhardt, G. Antar, and S. Zweben, Rev. Sci. Instrum. 72, 953 (2001).

[10] G. Andresen, W. Bertsche, P. D. Bowe, C. Bray, E. Butler, C. Cesar, S. Chapman, M. Charlton, S. S. El Nasr, J. Fajans, et al., Rev. Sci. Instrum. 80, 123701 (2009).

[11] A. Peurrung and J. Fajans, Rev. Sci. Instrum. 64, 52 (1993).

[12] K. Vanheusden, W. Warren, C. Seager, D. Tallant, J. Voigt, and B. Gnade, J. Appl. Phys 79, 7983 (1996). 


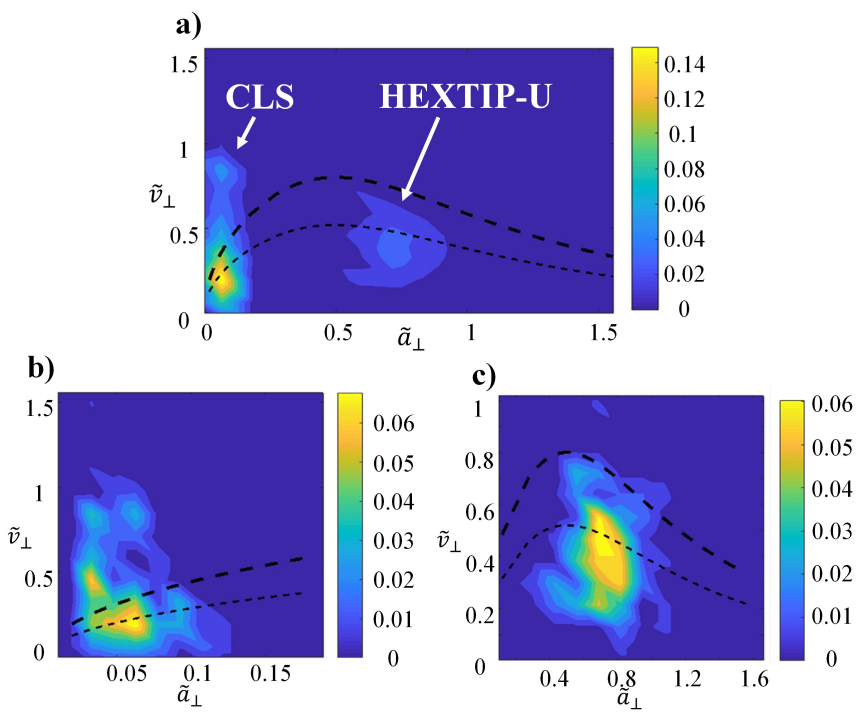

FIG. 10. Joint PDF of normalized radial blob velocity $\left(\tilde{v}_{\perp}\right)$ and vertical blob size $\left(\tilde{a}_{\perp}\right)$ of structures identified by the CLS and HEXTIP-U (a). Zoom on the PDF of b) structures identified by the CLS and c) structures identified by HEXTIP-U. The $\tilde{v}_{\perp}$ and $\tilde{a}_{\perp}$ of each structure are averaged on its lifetime. The black dashed lines represent the blob velocity scaling law with $\delta n / n=1$ (thick line) and $\delta n / n=0.65$ (thin line)
[13] T. Pedersen et al., Nat. Commun. 7, 13493 (2016).

[14] R. Jaenicke, E. Ascasibar, P. Grigull, I. Lakicevic, A. Weller, M. Zippe, H. Hailer, and K. Schworer, Nucl. Fusion 33, 687 (1993).

[15] D. A. D'Ippolito and J. R. Myra, Phys. Plasmas 10, 4029 (2003).

[16] M. Podestà, A. Fasoli, B. Labit, M. McGrath, S. H. Müller, and F. M. Poli, Plasma Phys. Control. Fusion 47, 1989 (2005).

[17] K. Gustafson, P. Ricci, A. Bovet, I. Furno, and A. Fasoli, Phys. Plasmas 19, 062306 (2012).

[18] M. Baquero-Ruiz, S. Alberti, O. Chellaï, I. Furno, T. Goodman, F. Manke, P. Micheletti, G. Plyushchev, and A. K. Skrivervik, Rev. Sci. Instrum. 89, 124702 (2018).

[19] I. Furno, C. Theiler, V. Chabloz, A. Fasoli, and J. Loizu, Phys. Plasmas 21, 012305 (2014).

[20] F. M. Poli, P. Ricci, A. Fasoli, and M. Podestà, Phys. Plasmas 15, 032104 (2008).

[21] C. Theiler, I. Furno, J. Loizu, and A. Fasoli, Phys. Rev. Lett. 108, 065005 (2012).

[22] C. Theiler, I. Furno, P. Ricci, A. Fasoli, B. Labit, S. H. Müller, and G. Plyushchev, Phys. Rev. Lett. 103, 065001 (2009).

[23] Phosphor P-24, ESPI Metals, Ashland OR, USA.

[24] M. Baquero Ruiz, O. Chellai, A. Fasoli, I. Furno, F. Manke, P. Ricci, P. Bowen, C. Morais, and W. Zhao, in Europhysics Conference Abstracts, CONF (2017) pp. P1-415.

[25] D. Iraji, I. Furno, A. Fasoli, and C. Theiler, Phys. Plasmas 17, 122304 (2010).

[26] N. Katz, J. Egedal, W. Fox, A. Le, and M. Porkolab, Phys. Rev. Lett. 101, 015003 (2008). 